Physics

Physics Research Publications

\title{
Localization-delocalization transition in a two-dimensional quantum percolation model
}

M. F. Islam

H. Nakanishi 


\title{
Localization-delocalization transition in a two-dimensional quantum percolation model
}

\author{
M Fhokrul Islam and Hisao Nakanishi \\ Department of Physics, Purdue University, West Lafayette, Indiana 47907, USA
}

(Received 6 March 2008; published 9 June 2008)

\begin{abstract}
We study the hopping transport of a quantum particle through randomly diluted percolation clusters in two dimensions realized both on the square and triangular lattices. We investigate the nature of localization of the particle by calculating the transmission coefficient as a function of energy $(-2<E<2$ in units of the hopping integral in the tight-binding Hamiltonian) and disorder, $q$ (probability that a given site of the lattice is not available to the particle). Our study based on finite-size scaling suggests the existence of delocalized states that depends on energy and the amount of disorder present in the system. For energies away from the band center $(E=0)$, delocalized states appear only at low disorder $(q<15 \%)$. The transmission near the band center is generally very small for any amount of disorder and therefore makes it difficult to locate the transition to delocalized states if any, but our study does indicate a behavior that is weaker than power-law localization. Apart from this localization-delocalization transition, we also find the existence of two different kinds of localization regimes depending on energy and the amount of disorder. For a given energy, states are exponentially localized for sufficiently high disorder. As the disorder decreases, states first show power-law localization before showing a delocalized behavior.
\end{abstract}

DOI: 10.1103/PhysRevE.77.061109

\section{INTRODUCTION}

Unlike a classical particle, the transport of a quantum particle through a system is greatly influenced by the interference and tunneling effects. While the availability of a spanning path is the sole criteria for the transmittance of a classical particle through a system, a quantum particle may exhibit zero or very low transmittance even for a completely ordered system depending on details such as the boundary condition or the energy of the particle [1]. The quantum percolation system that we have investigated here includes the interference effect but does not include the tunneling effect. We, thus, expect a higher connectivity in underlying geometry to be required for nonzero transmission compared to its classical counterpart.

A major motivation for studying such a system is the question of whether a localized-to-delocalized (or perhaps, metal-to-insulator) transition exists in a two-dimensional (2D) system. The Anderson model and the quantum percolation model are two of the more common theoretical models that are used to study the transport properties of disordered systems. While the literature on both models agree on the existence of such a transition in three dimensions [2-4], the same question for quantum percolation in two dimensions appears to have remained a subject of controversy for over two decades. Based on the one-parameter scaling theory of Abrahams et al. [5], it was widely believed that there can be no metal-to-insulator transition in 2D universally in the absence of a magnetic field or interactions for any amount of disorder. Moreover, the scaling theory predicts that all states are exponentially localized in a thermodynamic limit for any amount of disorder and therefore that no transition exists except at zero dilution. (However, see Goldenfeld and Haydock [6], which asserts the existence of a transition between two different kinds of localized regimes at a finite disorder in addition to a localized-delocalized transition at an infinitesimal dilution, even for the Anderson model in two dimensions.)

However, whether the scaling theory also applies to quantum percolation has been debated in recent years. Even re-
PACS number(s): 64.60.ah, 05.60.Gg

stricting attention to quantum percolation, which lacks many effects that are expected to play important roles in metalinsulator transitions, there is a long-standing controversy as to the presence or absence of an extended state and of a phase transition between the prevalent localized state and a more elusive extended state in two dimensions. On one hand, some studies such as those made using the $d$ log Padé approximation method [7], real space renormalization method [8], and the inverse participation ratio [9] found a transition from exponentially localized states to nonexponentially localized states for a range of site concentrations between $0.73 \leq p_{q} \leq 0.87$ on the square lattice. So did a study of energy level statistics [10], one of the spread of a wave packet initially localized at a site [11], and one of a transfer matrix [12], where the nature of the delocalized state remained not fully understood. On the other hand, studies such as the scaling work based on numerical calculation of the conductance $[13,14]$, the investigation of vibration-diffusion analogy [15], finite-size scaling analysis and transfer matrix methods [16], and vector recursion technique [17] found no evidence of a transition. A study by Inui et al. [18] found all states to be localized except for those with particle energies at the middle of the band and when the underlying lattice is bipartite, such as a square lattice. More recently, Cuansing and Nakanishi [19] used an approach first suggested by Daboul et al. [7] to calculate conductance directly for clusters of up to several hundred sites and, extrapolating those results by finite-size scaling, suggested that delocalized states exist and thus a transition would have to exist as well. The current paper extends the relevant portion of the latter work by studying much larger clusters, which has allowed considerably more detailed analyses.

In the mean time, experiments performed in the early 1980s on different 2D systems [20-22] confirmed the scaling theory predictions. However, a number of experiments on dilute low disordered Si MOSFET and GaAs/AlGaAs heterostructures that appeared more recently seem to suggest that a metallic state may be possible in two dimensions 


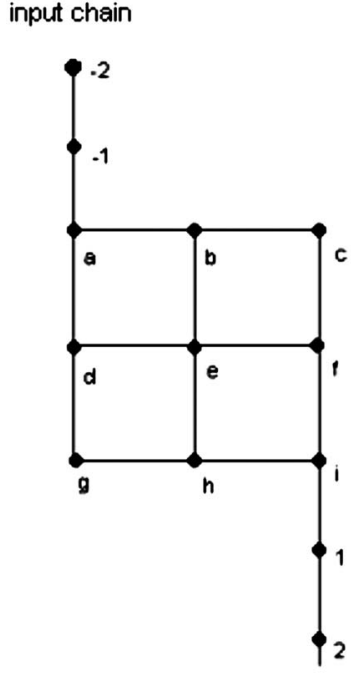

(a)

output chain

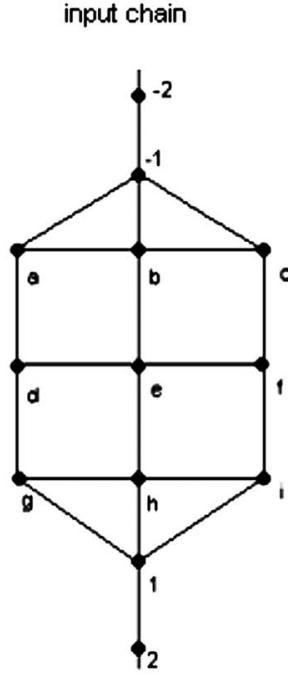

(b)

FIG. $1.3 \times 3$ square lattice: (a) point-to-point connection and (b) busbar-type connection. The letters label the lattice points of the cluster part of the Hamiltonian, while numbers label those of the leads. The same sequence of labeling is used for all sizes of the clusters in this work.

[23-26]. For reviews of these experiments, see Abrahams et al. [27], and references therein.

In this work, we do not address the issues of these experiments, but rather concentrate on the formally much simpler quantum percolation model, which has neither magnetic field nor interactions but contains binary disorder with infinite barriers at randomly diluted sites. Previously we have investigated the same problem using a dynamical approach where we have studied the properties of a disordered system by tracking how a quantum particle, described by a wave packet, propagates through the system [28]. In this paper we adopt a stationary state approach where we calculate the transmission characteristics by solving a time independent Schrödinger equation for a tight-binding Hamiltonian. In Sec. II we describe the model and the numerical approach used in this work. In Sec. III we discuss the numerical results and in Sec. IV we present the summary and conclusion of our study.

\section{QUANTUM PERCOLATION MODEL AND NUMERICAL APPROACH}

We study quantum percolation that is described by the Hamiltonian

$$
H=\sum_{\langle i j\rangle} V_{i j}|i\rangle\langle j|+\text { H.c. },
$$

where $|i\rangle$ and $|j\rangle$ are tight-binding basis functions at sites $i$ and $j$, respectively, and $V_{i j}$ is the hopping matrix element, which is equal to zero if $i$ and $j$ are not nearest neighbors. We have realized this model on both square and triangular lattices that can have at most four and six nearest neighbors, respectively. If the system is completely ordered, $V_{i j} \equiv V_{0}$ (uniform) and $V_{0}$ sets the overall energy scale, where we use
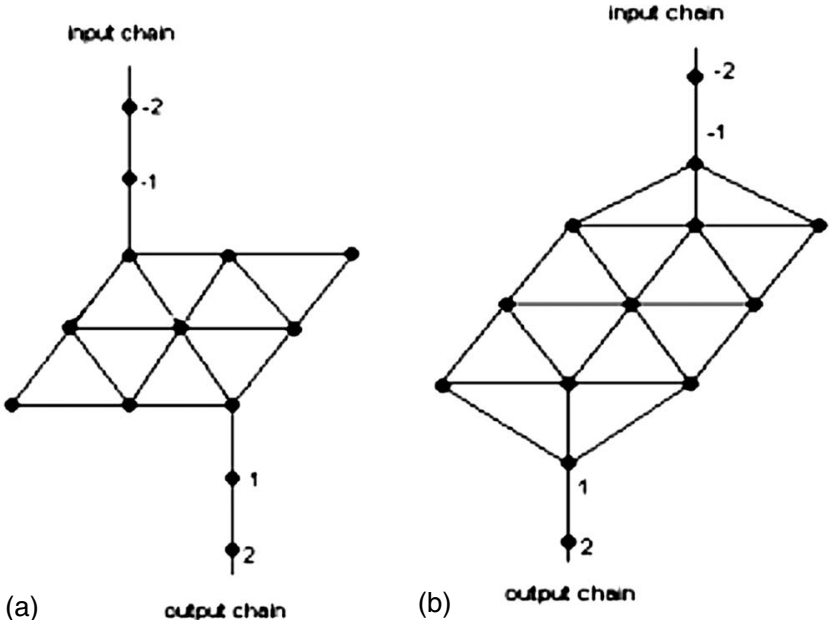

FIG. $2.3 \times 3$ triangular lattice: (a) point-to-point connection and (b) busbar-type connection.

$V_{0}=1$ as the nominal standard value. On the other hand, since in this work we are interested in transport through a disordered system, we will introduce random dilution by removing a fraction of sites from the lattice and set $V_{i j}=0$ for the bonds between the diluted sites and their neighbors. $V_{i j}$ $=0$ for nearest neighbors $i$ and $j$ means that an infinite barrier exists between the pair of sites.

To study the transmission of a quantum particle we connect two semi-infinite 1D leads, one as the input and the other as the output lead, to the $2 \mathrm{D}$ cluster. Although the system can be studied using different types of connection of the leads, in this study we only use a point-to-point type contact where the input lead is connected to only one lattice site on the input side edge of the cluster and the output lead is connected also to only one lattice site on the opposite edge of the cluster. Another possible connection type is the busbar-type contact, where all the lattice points on the input side edge of the cluster are connected to the input lead, while all the lattice points on the output side of the cluster are connected to the output lead. Figures 1 and 2 illustrate the connection of the leads for the square and the triangular lattices, respectively.

The wave function of the entire cluster-lead system can be calculated by solving the time-independent Schrödinger equation as follows:

$$
H \psi=E \psi,
$$

where

$$
\psi=\left(\begin{array}{c}
\psi_{\text {in }} \\
\psi_{\text {cluster }} \\
\psi_{\text {out }}
\end{array}\right),
$$

and $\psi_{\text {in }}=\left\{\psi_{-(n+1)}\right\}$, and $\psi_{\text {out }}=\left\{\psi_{+(n+1)}\right\}, n=0,1,2 \ldots$, are the input and output chain part of the wave function, respectively.

Since the leads are of infinite length, the matrix form of the Schrödinger equation [Eq. (2)] becomes an infinite-size problem. To reduce it to a numerically finite problem we use an ansatz proposed by Daboul et al. [7], which assumes that 
the input and output part of the wave function are of the form of plane waves as follows:

$$
\begin{gathered}
\psi_{\text {in }} \rightarrow \psi_{-(n+1)}=e^{-i n \kappa}+r e^{i n \kappa}, \\
\psi_{\text {out }} \rightarrow \psi_{+(n+1)}=t e^{i n \kappa},
\end{gathered}
$$

where $r$ is the amplitude of reflected wave, and $t$ is the amplitude of the transmitted wave. This ansatz is consistent with the Schrödinger equation only for the wave vector $\kappa$ that is related to the energy $E$ by

$$
E=e^{-i \kappa}+e^{i \kappa}
$$

Using this ansatz along with the energy restriction Eq. (4), the matrix equation for a $3 \times 3$ cluster connected to semiinfinite chains [Fig. 1(a)] reduces to (for details, see Ref. [7])

$$
\left(\begin{array}{rrrrrrrrrrr}
-E+e^{i \kappa} & c & 0 & 0 & 0 & 0 & 0 & 0 & 0 & 0 & 0 \\
c & -E & 1 & 0 & 1 & 0 & 0 & 0 & 0 & 0 & 0 \\
0 & 1 & -E & 1 & 1 & 1 & 0 & 0 & 0 & 0 & 0 \\
0 & 0 & 1 & -E & 0 & 0 & 1 & 0 & 0 & 0 & 0 \\
0 & 1 & 0 & 0 & -E & 1 & 0 & 1 & 0 & 0 & 0 \\
0 & 0 & 1 & 0 & 1 & -E & 1 & 0 & 1 & 0 & 0 \\
0 & 0 & 0 & 1 & 0 & 1 & -E & 0 & 0 & 1 & 0 \\
0 & 0 & 0 & 0 & 1 & 0 & 0 & -E & 1 & 0 & 0 \\
0 & 0 & 0 & 0 & 0 & 1 & 0 & 1 & -E & 1 & 0 \\
0 & 0 & 0 & 0 & 0 & 0 & 1 & 0 & 1 & -E & c \\
0 & 0 & 0 & 0 & 0 & 0 & 0 & 0 & 0 & c & -E+e^{i \kappa}
\end{array}\right)\left(\begin{array}{c}
1+r \\
\psi_{1} \\
\psi_{2} \\
\psi_{3} \\
\psi_{4} \\
\psi_{5} \\
\psi_{6} \\
\psi_{7} \\
\psi_{8} \\
\psi_{9} \\
t
\end{array}\right)=\left(\begin{array}{c}
e^{i \kappa}-e^{-i \kappa} \\
0 \\
0 \\
0 \\
0 \\
0 \\
0 \\
0 \\
0 \\
0 \\
0
\end{array}\right)
$$

Here $c$ is the coupling of the leads with the cluster and its value set to 1 for all the calculations done in this work. The effect of $c \neq 1$ on transmission is discussed in one of our previous works [29]. The busbar configuration of Fig. 1(b) has a similar expression.

Equation (5) is the exact expression for a $2 \mathrm{D}$ system connected to semi-infinite chains with continuous eigenvalues ranging between -2 and +2 . The spectrum is continuous because it is still effectively infinite and it is nondegenerate except for the reversal of left and right.

The main advantage of using this ansatz is that it not only allows us to calculate the wave function but also helps us to study the transmission characteristics of the corresponding state directly. The transmission and the reflection coefficients are obtained by taking the absolute square of $t$ and $r$, respectively, i.e., $T=|t|^{2}$ and $R=|r|^{2}$. A disadvantage, on the other hand, is that Eq. (4), which relates the wave vector of the incident particle with the energy, restricts the energy of the particle to between -2 and +2 . This restricts our ability to study the system in the whole possible energy range since for our system the energy could, in principle, range between -4 and +4 . This is due, of course, to the effectively onedimensional nature of the system forced by attaching $1 \mathrm{D}$ semi-infinite leads and by looking at plane waves spreading over the entire leads.

\section{NUMERICAL RESULTS}

The model that we are using to calculate transmission has two adjustable parameters, namely, the energy of the particle,
$E$ and the amount of disorder present in the system, $q$ (probability that a given site is not available to the hopping particle). To study the presence or absence of a localizationdelocalization transition, one needs to investigate the behavior of the 2D system in the thermodynamic limit. This, however, is not possible in numerical methods, and therefore, we resort to a finite-size scaling approach in which we calculate the transmission while gradually increasing the size of the system for a given amount of disorder. The result is then extrapolated to study the bulk behavior in the thermodynamic limit.

Though there are many combinations possible for connecting the input and the output leads with the cluster, for the work that follows, the leads are connected diagonally with the clusters, since this arrangement generally gives higher transmission. In addition to that, to minimize the effect of the boundary on the interior property of the disordered clusters, we made good contacts by keeping the nine sites nearest to both the input and the output contact points always occupied (that is, available to the hopping particle). We calculate the transmission as a function of the system size for many levels of disorder and at different energies. The general trend for all the transmission curves at different energies are similar, so we will discuss here only two energies, one that is away from the band center and one very close to the band center.

\section{A. Energies away from the band center}

We first study the transmission at energy $E=1.6$ for different levels of disorder. For each level of disorder, we have 
TABLE I. Table for fitting parameters at $E=1.6$. Shown in parentheses are the lower and upper bounds for a $95 \%$ confidence level.

\begin{tabular}{ccccc}
\hline \hline \multirow{2}{*}{$\begin{array}{c}\text { Fit } \\
\%)\end{array}$} & $\begin{array}{c}c \\
\text { equation }\end{array}$ & \multirow{2}{*}{$R_{\sigma}^{2}$} \\
\cline { 3 - 4 } & & $a$ & \\
\hline 5 & & $0.78(0.69,0.89)$ & $0.26(0.23,0.30)$ & 0.95 \\
10 & & $1.09(0.89,1.32)$ & $0.44(0.39,0.50)$ & 0.96 \\
13 & & $1.24(1.01,1.52)$ & $0.54(0.49,0.60)$ & 0.97 \\
15 & $T=a L^{-b}$ & $1.404(1.16,1.70)$ & $0.63(0.58,0.68)$ & 0.98 \\
20 & & $2.70(2.25,3.25)$ & $0.96(0.91,1.01)$ & 0.99 \\
23 & & $4.08(3.47,4.81)$ & $1.19(1.15,1.23)$ & 0.99 \\
25 & & $11.3(6.20,20.8)$ & $1.59(1.43,1.75)$ & 0.97 \\
26 & & $16.3(8.98,29.5)$ & $1.77(1.61,1.93)$ & 0.97 \\
28 & & $36.2(18.4,71.5)$ & $2.12(1.93,2.30)$ & 0.98 \\
30 & & $0.18(0.12,0.25)$ & $0.06(0.05,0.06)$ & 0.97 \\
35 & $T=a e^{-b L}$ & $0.24(0.12,0.46)$ & $0.12(0.11,0.13)$ & 0.97 \\
38 & & $0.96(0.15,6.12)$ & $0.22(0.19,0.26)$ & 0.94 \\
\hline \hline
\end{tabular}

calculated the transmission by gradually increasing the size of the clusters from $10 \times 10$ to a maximum size of 180 $\times 180$. We have randomly generated one thousand clusters of a given size for each level of disorder and average transmission is calculated for each size of the cluster and each level of disorder. The log-log plot of transmission against the size of the clusters at $E=1.6$ is shown for various disorder levels in Fig. 3.

We observe from Fig. 3 that at lower disorder, transmission decreases almost linearly in the log-log plot as the size of the clusters increases, suggesting a power-law behavior of

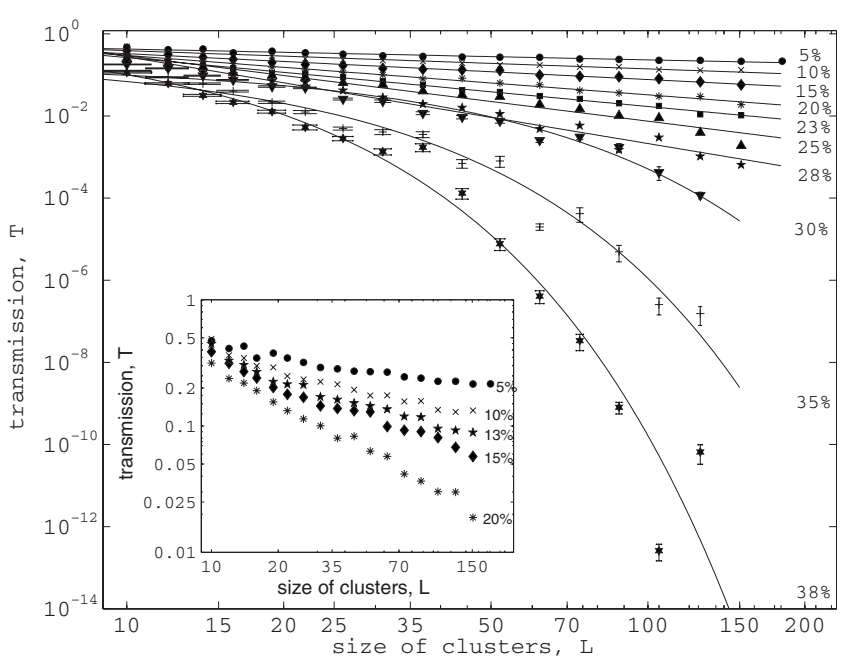

FIG. 3. The log-log plot of transmission through disordered clusters at $E=1.6$. Each data point is the average over 1000 independent realizations. The transmission curves for 5\%, 10\%, 13\%, $15 \%$, and $20 \%$ disorders are separately shown in the inset.

transmission in linear scale. This trend continues until disorder $q$ increases to about $28 \%$. Above that, transmission falls exponentially as is evident from the lowest three curves in Fig. 3. We have fitted the data both to power laws and exponentials and the best fit forms along with the corresponding fitting parameters and coefficient of regression $R_{\sigma}^{2}$, for different levels of disorder, are presented in Table I. Although there are many data points that appear to be several $\sigma$ 's from the best fit exponentials particularly for higher disorder, they are believed to be due to the discrete and loose-packed structure of the lattice and not due to some unknown systematic errors. (The transmission curves of the $13 \%$ and $26 \%$ disor-

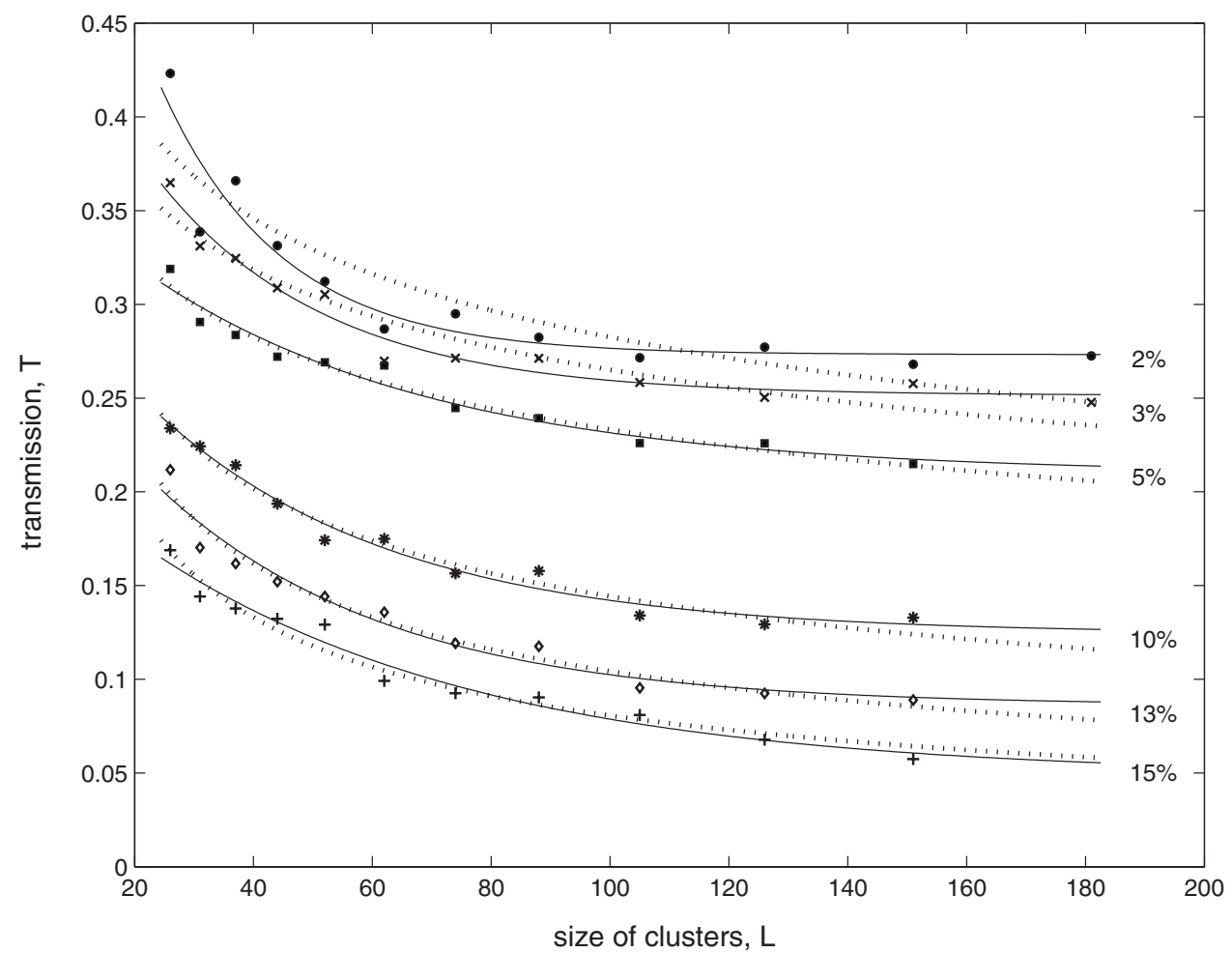

FIG. 4. The linear plot of transmission through clusters with $2 \%, 3 \%, 5 \%, 10 \%, 13 \%$, and $15 \%$ disorders at $E=1.6$ for larger sizes of the clusters $(L \geq 25)$. Each data point is the average over 1000 independent realizations. The dotted lines represent power-law fits and the solid lines represent the exponential fits with offset. 
TABLE II. Table for fitting parameters of the linear plot at $E=1.6$. Shown in parentheses are the lower and upper bounds for a $95 \%$ confidence level.

\begin{tabular}{|c|c|c|c|c|c|c|c|c|c|}
\hline \multirow{2}{*}{$\begin{array}{c}q \\
(\%)\end{array}$} & \multicolumn{4}{|c|}{$T=a L^{-b}$} & \multicolumn{5}{|c|}{$T=a e^{-b L}+c$} \\
\hline & $a$ & $b$ & $R_{\sigma}^{2}$ & SSE & $a$ & $b$ & $c$ & $R_{\sigma}^{2}$ & SSE \\
\hline 2 & 0.78 & 0.22 & 0.79 & $5 \times 10^{-3}$ & 0.48 & 0.05 & $\begin{array}{c}0.27 \\
(0.26,0.29)\end{array}$ & 0.89 & $2 \times 10^{-3}$ \\
\hline 3 & 0.67 & 0.20 & 0.91 & $1 \times 10^{-3}$ & 0.27 & 0.03 & $\begin{array}{c}0.25 \\
(0.24,0.26)\end{array}$ & 0.96 & $5 \times 10^{-4}$ \\
\hline 5 & 0.61 & 0.21 & 0.96 & $3 \times 10^{-4}$ & 0.17 & 0.02 & $\begin{array}{c}0.21 \\
(0.19,0.23)\end{array}$ & 0.95 & $4 \times 10^{-4}$ \\
\hline 10 & 0.78 & 0.37 & 0.97 & $3 \times 10^{-4}$ & 0.21 & 0.02 & $\begin{array}{c}0.12 \\
(0.11,0.14)\end{array}$ & 0.97 & $3 \times 10^{-4}$ \\
\hline 13 & 0.95 & 0.48 & 0.96 & $5 \times 10^{-4}$ & 0.22 & 0.03 & $\begin{array}{c}0.09 \\
(0.06,0.1)\end{array}$ & 0.94 & $6 \times 10^{-4}$ \\
\hline 15 & 1.00 & 0.55 & 0.96 & $4 \times 10^{-4}$ & 0.18 & 0.02 & $\begin{array}{c}0.05 \\
(0.02,0.08)\end{array}$ & 0.96 & $4 \times 10^{-4}$ \\
\hline
\end{tabular}

der are not shown in Fig. 3 since they are very close to the nearby transmission curves).

It should be noted that the curve fitting is performed in $\log -\log$ scale and then converted to linear scale along with the parameters. Thus to fit the data, say for $q=15 \%$, we first calculate the logarithm of the size of the cluster and the corresponding transmission, i.e., $x=\log (L)$ and $y=\log (T)$. We then fit these logarithmic data with the curve $y=m \cdot x+c$, and converted the result back to linear scale.

Because of the large differences in transmission for different disorder, we used the logarithmic scale for transmission in Fig. 3, which in turn makes it difficult to see the finer details of the transmission curve, particularly at lower disorders. For instance, Fig. 3 appears to suggest an excellent linear fit at disorders below 15\%. To investigate more closely the nature of transmission at lower disorder we have plotted

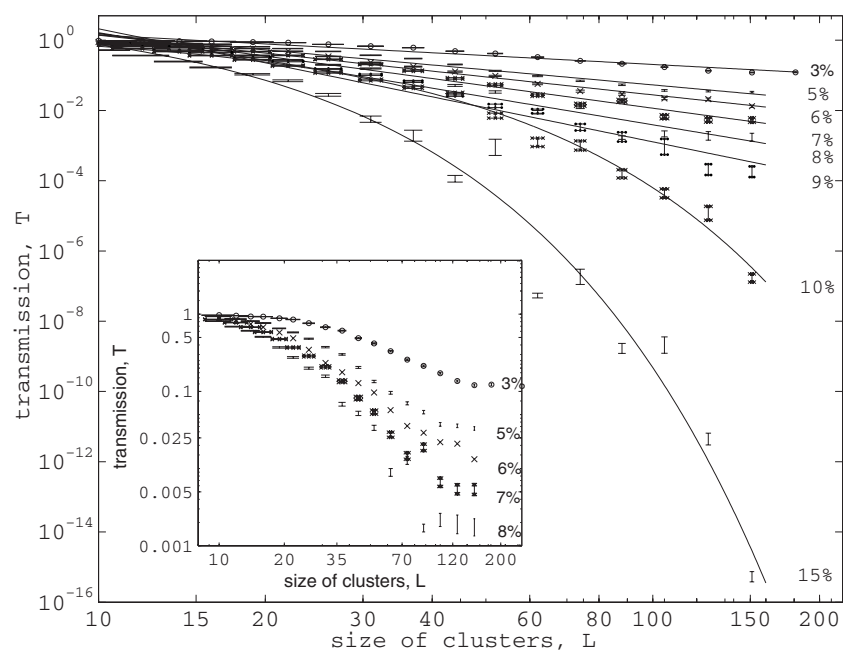

FIG. 5. The log-log plot of transmission through disordered clusters at $E=0.001$. Each data point is the average over 1000 independent realizations. The transmission curves for $5 \%, 6 \%, 7 \%$, and $8 \%$ disorders are also separately shown in the inset. the first five curves of Table I separately in the inset of Fig. 3. It is evident from the inset that transmission decreases much more slowly than a power law at larger values of $L$ for disorder up to about $13 \%$. Above $13 \%$ power laws do appear to give good fits of the data for the entire range of cluster sizes used. To investigate this lower disorder regime further, we have obtained the transmission for $2 \%$ and $3 \%$ disorder and have plotted these data along with those for $5 \%-15 \%$ disorder in a linear graph as shown in Fig. 4 for larger clusters ( $L \geq 25$ ). Each data set is fitted with two different curves, namely [30], a power law

$$
T=a L^{-b},
$$

and an exponential with offset,

$$
T=a e^{-b L}+c .
$$

The dotted and solid lines represent fits with Eqs. (6) and (7), respectively. As we can see from Fig. 4, exponential with offset, $c$, gives significantly better visual fits for the data compared to power laws at least for $2 \%$ and $3 \%$ disorder,

TABLE III. Table for fitting parameters at $E=0.001$. Shown in parentheses are the lower and upper bounds for a $95 \%$ confidence level.

\begin{tabular}{ccccc}
\hline \hline \multirow{2}{*}{$\begin{array}{c}\text { Fit } \\
(\%)\end{array}$} & $\begin{array}{c}\text { Fit } \\
\text { equation }\end{array}$ & \multirow{2}{*}{$R_{\sigma}^{2}$} \\
\cline { 3 - 4 } & & $a$ & $b$ & \\
\hline 5 & & $38.2(22.7,64.5)$ & $1.43(1.29,1.57)$ & 0.97 \\
6 & & $70.0(69.9,113)$ & $1.70(1.57,1.83)$ & 0.98 \\
7 & $T=a L^{-b}$ & $204(97.6,429)$ & $2.12(1.93,2.32)$ & 0.97 \\
8 & & $691(216,2206)$ & $2.62(2.31,2.93)$ & 0.96 \\
9 & & $0.85(0.53,1.35)$ & $0.06(0.06,0.07)$ & 0.96 \\
10 & $T=a e^{-b L}$ & $1.88(1.31,2.69)$ & $0.10(0.11,0.10)$ & 0.99 \\
15 & & $8.00(1.90,33.7)$ & $0.24(0.21,0.26)$ & 0.97 \\
\hline \hline
\end{tabular}




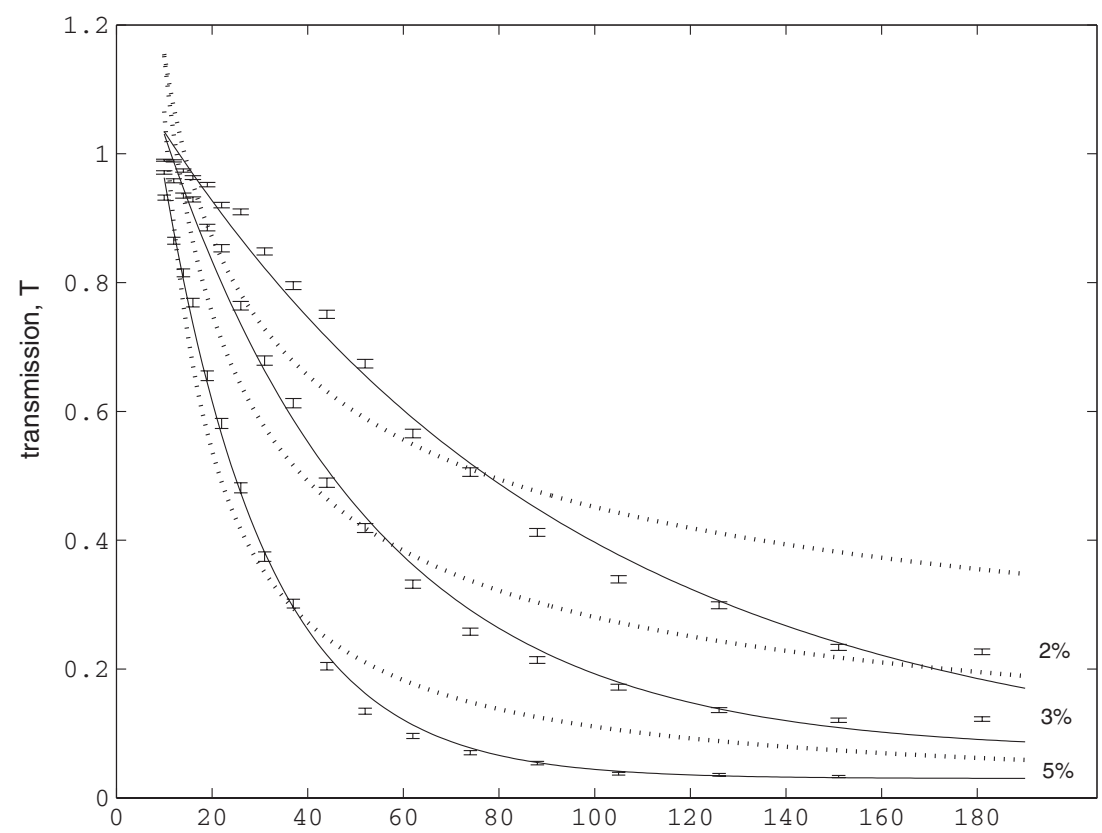

(a)

size of clusters, $L$

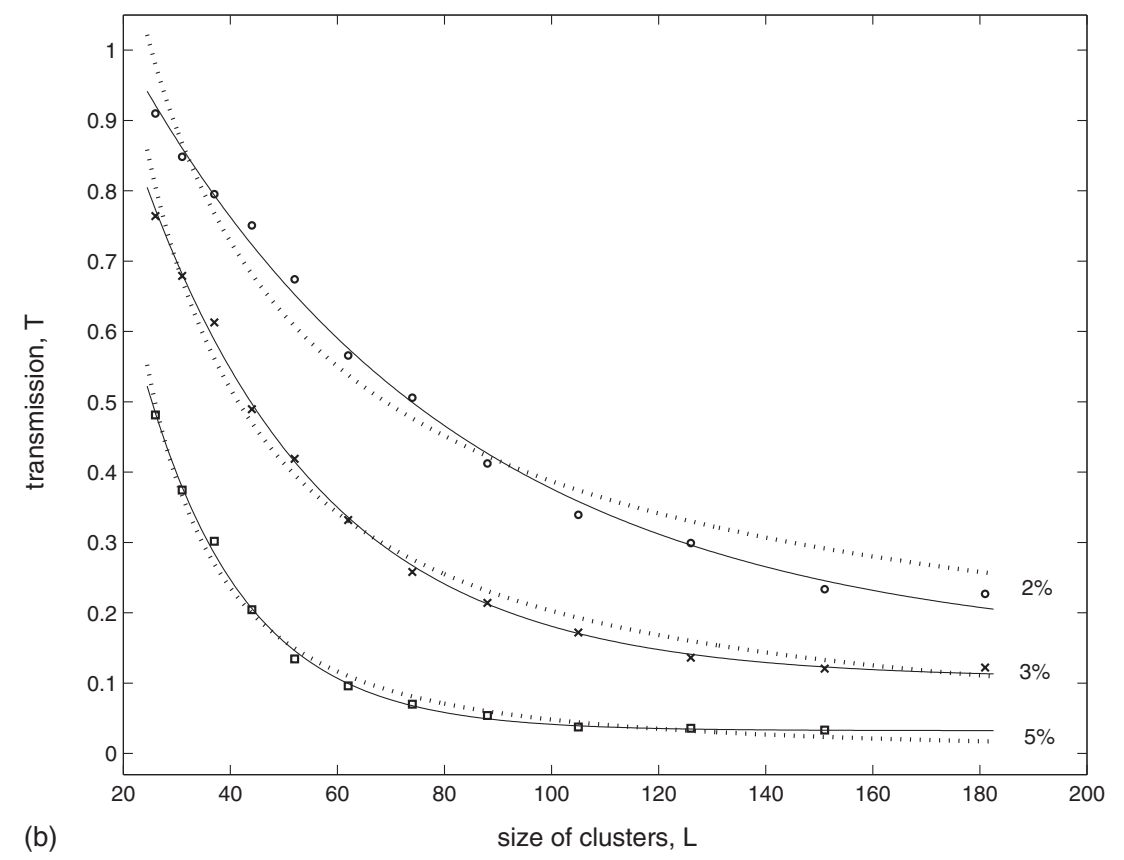

FIG. 6. The linear plot of transmission through clusters with $2 \%, 3 \%$, and $5 \%$ disorder at $E$ $=0.001$. (a) Plot with all data points and (b) plot with data points only for larger sizes of the clusters. Each data point is the average over 1000 independent realizations. The dotted lines represent the power-law fits. while the goodness of fits for higher disorders appear to be a toss-up.

To compare the goodness of fits we need to consider not only the visual fits, but the values of $R_{\sigma}^{2}$ and the sum square error (SSE) as well, and it is evident from Table II and Fig. 4(b) that an exponential with offset gives significantly better fits than a power law, except for $15 \%$ disorder, at which both fits are quite close.

The above study, thus, suggests the existence of three regimes of transmission. At higher disorder transmission drops exponentially as the size of clusters increases. At intermediate disorder, transmission follows power law, whereas at low disorder transmission approaches a constant offset $c$, suggesting possible delocalization of the states. The value of the offset, however, depends on energy. For a given disorder, $c$ decreases as the energy increases, except for energies very close to the band center where transmission itself is very low.

\section{B. Energies near the band center}

In this subsection we discuss the nature of transmission at an energy near the band center. We investigate the scaling behavior at $E=0.001$ for different levels of disorder. The $\log$-log plot of transmission as a function of system size is shown in Fig. 5.

At this energy, transmission decreases exponentially for disorder as low as $9 \%$ as is evident from Fig. 5. Below $9 \%$ the transmission curves appear to fit well with power laws. 
TABLE IV. Table for fitting parameters of the linear plot at $E=0.001$. Shown in parentheses are the lower and upper bounds for a $95 \%$ confidence level.

\begin{tabular}{|c|c|c|c|c|c|c|c|c|c|}
\hline \multirow{2}{*}{$\begin{array}{c}q \\
(\%)\end{array}$} & \multicolumn{4}{|c|}{$T=a L^{-b}$} & \multicolumn{5}{|c|}{$T=a e^{-b L}+c$} \\
\hline & $a$ & $b$ & $R_{\sigma}^{2}$ & SSE & $a$ & $b$ & $c$ & $R_{\sigma}^{2}$ & SSE \\
\hline 2 & 9.25 & 0.69 & 0.96 & $2 \times 10^{-2}$ & 1.19 & 0.02 & $\begin{array}{c}0.15 \\
(0.09,0.20)\end{array}$ & 0.99 & $3 \times 10^{-3}$ \\
\hline 3 & 22.7 & 1.03 & 0.99 & $7 \times 10^{-3}$ & 1.44 & 0.03 & $\begin{array}{c}0.11 \\
(0.09,0.12)\end{array}$ & 0.99 & $1 \times 10^{-3}$ \\
\hline 5 & 141 & 1.73 & 0.99 & $2 \times 10^{-3}$ & 1.79 & 0.05 & $\begin{array}{c}0.03 \\
(0.02,0.04)\end{array}$ & 0.99 & $5 \times 10^{-4}$ \\
\hline 6 & 198 & 1.95 & 0.99 & $4 \times 10^{-4}$ & 1.53 & 0.06 & $\begin{array}{c}0.02 \\
(0.01,0.03)\end{array}$ & 0.99 & $7 \times 10^{-4}$ \\
\hline
\end{tabular}

The fitting parameters of the different curves are tabulated in Table III along with the coefficient of regression, $R_{\sigma}^{2}$.

The $R_{\sigma}^{2}$ values for $5 \%, 6 \%, 7 \%$, and $8 \%$ disorder from Table III suggest a good fit of these data with power laws. However, if we take a closer look at the transmission curves for these disorders in the inset, we observe a significant deviation from straight lines as the size of the cluster increases. It is clear that at lower disorders, the falling trend in transmission is much slower than the power law, similar to what we have observed at energies far from the band center. However, transmission is generally much smaller for the same amount of disorder compared with the case of energies away from the band center.

As before, we have obtained two more transmission data for $2 \%$ and $3 \%$ disorder and the results are plotted in Fig. 6 (a) along with that for 5\% disorder (the transmission curve for $6 \%$ disorder is too close to that of $5 \%$ disorder and is not shown in the figure). We show in Fig. 6(a) transmission data for the entire range of cluster sizes. We have fitted these data with both a power law and an exponential with offset as before, and the best fit curves along with the fit parameters are tabulated in Table IV. The dotted lines represent the power-law fits, and the solid lines represent exponential fits with offset. We notice that at this energy the best fit curve for each of the disorder amounts shown is clearly an exponential with offset rather than a power law. For a more direct comparison with Fig. 4, where we considered only the larger clusters $(L \geq 25)$, we also show the corresponding figure and fits for the same range of cluster sizes in Fig. 6(b) as well. In the latter figure, the difference in goodness of fits between power-law fits and exponential fits with offset is not as large, but it is still clear that the latter fits the data better.

The issue of localization length $L_{l}$, must also be discussed. Our finite-size scaling approach does not rely on any single system size but rather focuses on the trend as it increases. In fact, this analysis detects different trends depending on the dilution amount and we can estimate the localization length from the data themselves, at least in the clearly observed exponentially localized regime. Table V summarizes the simulation estimates of $L_{l}$ at different energies in the regions of disorder where the respective exponential fits $\left(a e^{-b L}\right)$ are significantly better than other types of fits. The localization length in the exponential regime can be estimated from $L_{l} \sim b^{-1}$. It is evident that most of our system sizes are sufficiently large compared with these estimates of $L_{l}$ and thus our results are internally consistent with exponential localization at these amounts of disorder.

For completeness we present here the scaling result for triangular lattice only for energy $E=1$ for different disorder. The curves appear very similar to those for square lattice as

TABLE V. Estimated values of the localization lengths from $T=a e^{-b L}$, localization length being $b^{-1}$.

\begin{tabular}{|c|c|c|c|c|c|c|c|}
\hline \multirow{2}{*}{$\begin{array}{c}q \\
(\%)\end{array}$} & \multicolumn{7}{|c|}{ Localization length (in units of lattice constant) } \\
\hline & $E=0.001$ & $E=0.05$ & $E=0.5$ & $E=1.0$ & $E=1.2$ & $E=1.6$ & $E=1.9$ \\
\hline 9 & 15.6 & & & & & & \\
\hline 10 & 9.7 & & & & & & \\
\hline 15 & 4.5 & & & & & & \\
\hline 20 & 2.7 & 16.7 & & & & & \\
\hline 25 & & 6.3 & & & & & 27.0 \\
\hline 30 & & & & 19.3 & & 17.2 & 13.3 \\
\hline 31 & & & 13.9 & & 16.7 & & \\
\hline 32 & & & 13.9 & & & & \\
\hline 35 & & & 8.8 & 6.7 & 7.1 & 8.2 & 5.4 \\
\hline 38 & & & 4.8 & 4.3 & 4.1 & 4.4 & \\
\hline
\end{tabular}




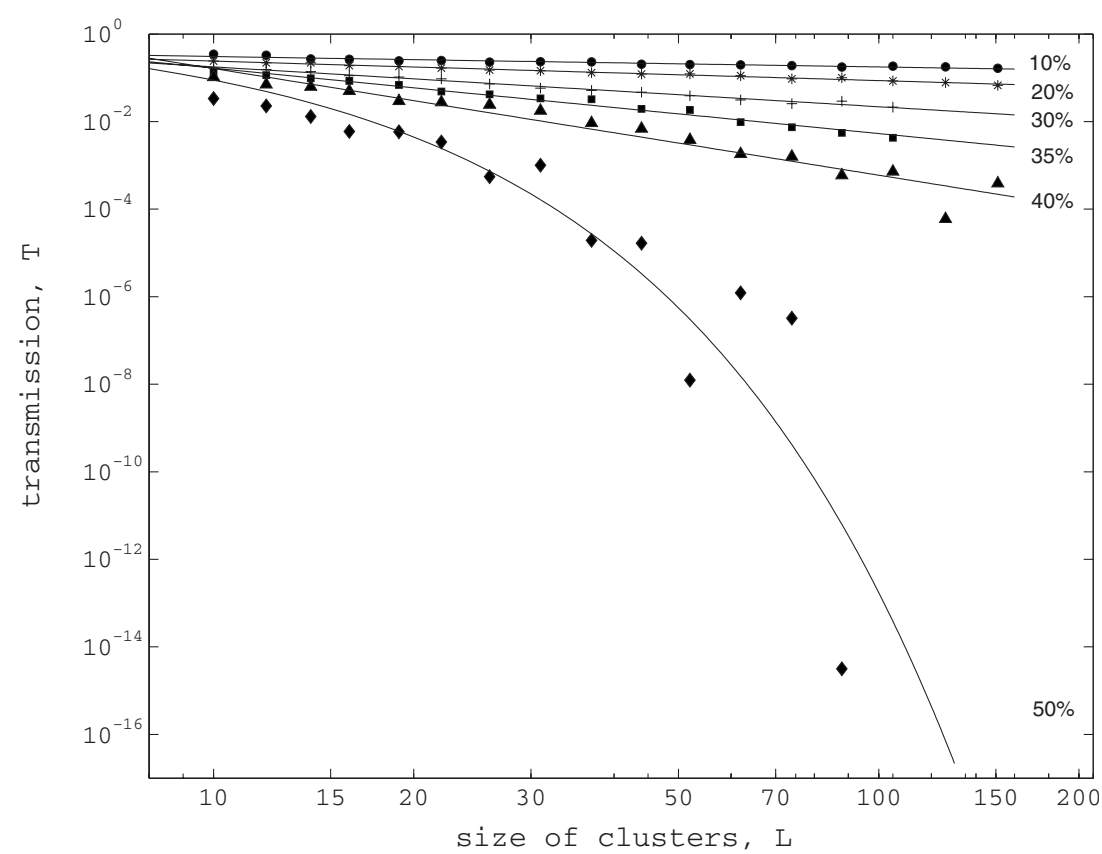

is evident from Fig. 7 except that the exponentially localized regime appears at a higher disorder. The result is expected since triangular lattice has six nearest neighbors and therefore requires more disorder to reduce the transmission.

All the numerical calculations in this work were based on finite clusters with the largest one being $180 \times 180$. Although our analysis based on finite-size scaling shows the appearance of delocalized states at low disorders, one needs to be cautious that at this regime localization length might be much larger than the system size. Our work also shows that the delocalized states at the band center appear only at very low disorders even though particle velocity is maximum around the band center for the 1D portions of the system. However, transmission depends in a complicated manner on the wave function inside the cluster portion and evidently the velocity in the chain portions alone does not determine it. In fact, the mean size of the eigenmodes is not monotone in energy and it is actually depressed near the band center, particularly for higher disorder.

TABLE VI. Classification of our data into different regimes in a 2D disordered system. ${ }^{*}$ indicates a crude estimate that could be off by a few $\%$.

\begin{tabular}{cccc}
\hline \hline Energy & \multicolumn{3}{c}{ Range of disorder } \\
\cline { 2 - 4 } & $\begin{array}{c}\text { Delocalized } \\
\text { states } \\
\text { regime }\end{array}$ & $\begin{array}{c}\text { Power law } \\
\text { localization } \\
\text { regime }\end{array}$ & $\begin{array}{c}\text { Exponential } \\
\text { localization } \\
\text { regime }\end{array}$ \\
\hline 0.001 & $0-6 \%^{*}$ & $7 \%-9 \%$ & $\geq 10 \%$ \\
0.05 & $0-12 \%^{*}$ & $15 \%-18 \%$ & $\geq 20 \%$ \\
0.5 & $0-10 \%^{*}$ & $15 \%-31 \%$ & $\geq 32 \%$ \\
1.0 & $0-10 \%^{*}$ & $15 \%-28 \%$ & $\geq 30 \%$ \\
1.2 & $0-10 \%^{*}$ & $15 \%-30 \%$ & $\geq 31 \%$ \\
1.6 & $0-13 \%^{*}$ & $15 \%-28 \%$ & $\geq 30 \%$ \\
1.9 & $0-5 \%^{*}$ & $10 \%-23 \%$ & $\geq 25 \%$ \\
\hline \hline
\end{tabular}

\section{SUMMARY AND CONCLUSION}

In this paper we have studied the behavior of a quantum particle in two-dimensional disordered clusters in a quantum percolation model. Our approach is based on calculating the transmission of the particle that enters into the cluster through a one-dimensional lead at one side of the cluster and exit through another lead at the other side of the cluster.

Our study based on finite-size scaling suggests the existence of three different regimes, depending on disorder. The range of these regimes, however, depends on the energy of the particle. Although we have shown in previous sections the results from two representative energies $(E=1.6$ that is far from the band center and $E=0.001$ that is very close to it), we have in fact obtained and analyzed the data for many more values of the energy. Table VI shows our approximate estimates of the range of disorder for which these regimes exist as the energy is varied.

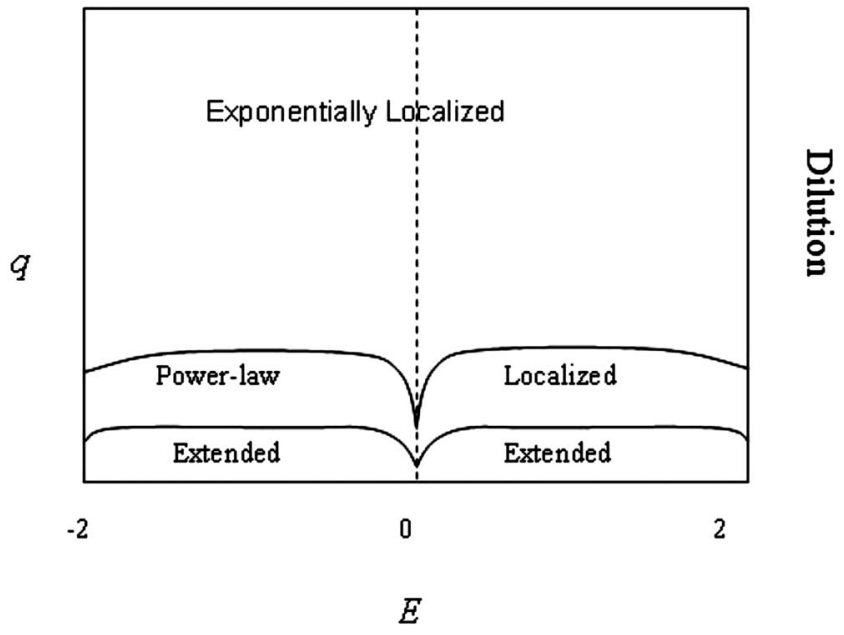

FIG. 8. A crude sketch of the three localization regimes in the space of energy $E$, and disorder $q$ based on the results shown in Table VI. 
Thus, our study suggests that at lower disorder, states are delocalized, contrary to the one-parameter scaling theory of Abrahams et al. [5]. In addition to delocalized transition, we observe a second kind of phase transition between the power law to exponentially localized regimes. A crude sketch of the phase diagram of a 2D disordered system based on our calculation is shown in Fig. 8.

For all energies considered in this work, except for those close to $E=0$, the delocalized states appear if the disorder is less than about $15 \%$. Above $15 \%$, there exist two different localization regimes. For intermediate energies $(0.5<E$ $<1.6$ ), states of the particle show a weaker form of localization characterized by a power-law size dependence of transmission for the range of disorder between $15 \%$ and $\sim 30 \%$. Above this range all states are localized exponentially. For energies close to $E=2$, the width of this power-law localization regime is reduced and states become exponentially localized even for $25 \%$ disorder.
The transmission near the band center, however, differs in some important ways compared to that at other energies. At $E \sim 0$, transmission is very low in the thermodynamic limit even for very low disorder. Though it is difficult to locate the delocalization transition because of the low transmission, our finite-size analysis does show a behavior much weaker than power-law localization (the inset of Fig. 5). The width of the power-law localization regime is very small and exponentially localized states appear for disorder as low as $9 \%$.

\section{ACKNOWLEDGMENTS}

We would like to thank A. Overhauser, Y. Lyanda-Geller, S. Savikhin, and many others for discussions, and the Physics Computer Network (PCN) at Purdue University where most of the numerical work was performed.
[1] E. Cuansing and Hisao Nakanishi, Phys. Rev. E, 70, 066142 (2004).

[2] C. M. Soukoulis, E. N. Economou, and G. S. Grest, Phys. Rev. B 36, 8649 (1987).

[3] Th. Koslowski and W. von Niessen, Phys. Rev. B 44, 9926 (1991).

[4] R. Berkovits and Y. Avishai, Phys. Rev. B 53, R16125 (1996).

[5] E. Abrahams, P. W. Anderson, D. C. Licciardello, and T. V. Ramakrishnan, Phys. Rev. Lett. 42, 673 (1979).

[6] N. Goldenfeld and R. Haydock, Phys. Rev. B 73, 045118 (2006).

[7] D. Daboul, I. Chang, and A. Aharony, Eur. Phys. J. B 16, 303 (2000).

[8] T. Odagaki and K. C. Chang, Phys. Rev. B 30, 1612 (1984).

[9] V. Srivastava and M. Chaturvedi, Phys. Rev. B 30, 2238 (1984).

[10] M. Letz and K. Ziegler, Philos. Mag. B 79, 491 (1999).

[11] H. N. Nazareno, P. E. de Brito, and E. S. Rodrigues, Phys. Rev. B 66, 012205 (2002).

[12] A. Eilmes, R. A. Römer, and M. Schreiber, Physica B (Amsterdam) 296, 46 (2001).

[13] G. Hałdaś, A. Kolek, and A. W. Stadler, Phys. Status Solidi B 230, 249 (2002).

[14] Y. Avishai and J. M. Luck, Phys. Rev. B 45, 1074 (1992).

[15] A. Bunde, J. W. Kantelhardt, and L. Schweitzer, Ann. Phys. 7, 372 (1998).

[16] C. M. Soukoulis and G. S. Grest, Phys. Rev. B 44, 4685 (1991).

[17] A. Mookerjee, I. Dasgupta, and T. Saha, Int. J. Mod. Phys. B 9, 2989 (1995).

[18] M. Inui, S. A. Trugman, and E. Abrahams, Phys. Rev. B 49, 3190 (1994).

[19] E. Cuansing and Hisao Nakanishi, Physica A, 387, 806 (2008).

[20] G. J. Dolan and D. D. Osheroff, Phys. Rev. Lett. 43, 721 (1979).

[21] D. J. Bishop, D. C. Tsui, and R. C. Dynes, Phys. Rev. Lett. 44,
1153 (1980).

[22] M. J. Uren, R. A. Davies, and M. Papper, J. Phys. C 13, L985 (1980).

[23] S. V. Kravchenko, G. V. Kravchenko, J. E. Furneaux, V. M. Pudalov, and M. D'Iorio, Phys. Rev. B 50, 8039 (1994).

[24] S. V. Kravchenko, W. E. Mason, G. E. Bowker, J. E. Furneaux, V. M. Pudalov, and M. D'Iorio, Phys. Rev. B 51, 7038 (1995).

[25] M. P. Sarachik and S. V. Kravchenko, Proc. Natl. Acad. Sci. U.S.A. 96, 5900 (1999).

[26] M. P. Sarachik, in More is Different: Fifty Years of Condensed Matter Physics, edited by N. P. Ong and R. N. Bhatt (Princeton University Press, Princeton, NJ, 2001), pp. 42-43.

[27] E. Abrahams, S. V. Kravchenko, and M. P. Sarachik, Rev. Mod. Phys. 73, 251 (2001).

[28] Md Fhokrul Islam and Hisao Nakanishi (unpublished).

[29] Md Fhokrul Islam and Hisao Nakanishi (unpublished).

[30] For exponential and power laws, the motivation comes from a picture where the transmission arises from overlapping wave functions. Then the overlap integral will decay exponentially with the distance if the wave functions are themselves exponential. It will decay by a power law if they are power-law or long-ranged. The motivation for fitting to an exponential plus a constant offset to test for delocalization can be understood by a simple analogy to, say, the correlation function of an Ising model below $T_{c}$. If there were finite order (transmission, here), then there would be a constant offset. Except for this "order", the correlation should behave very similarly to the "disordered" phase since the critical correlation of the fluctuations should only occur at $T c$ and not for $T<T_{c}$ or $T>T_{c}$. That is, if we subtract the offset, the remainder should decay the same way as it does above $T_{c}$, i.e., exponentially. Of course, in our problem, this analogy may not be true and some sort of residual long-range correlations may still exist after subtracting the offset, which might require a power-law plus offset instead. However, our first guess would be to use an exponential plus offset. 